\title{
Diabetes Technology and the Human Factor
}

\author{
Alon Liberman ${ }^{1}$ and Bruce Buckingham ${ }^{2}$
}

\section{Introduction}

TN RECENT YEARS significant progress has been made in diabetes technologies. Diabetes technologies such as continuous subcutaneous insulin infusion (CSII) and continuous glucose monitoring (CGM) play a significant part of diabetes treatment, and there have been many studies that emphasize the advantages of using these technologies.

The scientific field of "human factors" uses information from a variety of disciplines, including psychology, engineering, industrial design, statistics, biomechanics, ethnography, and physiology and focuses on people and their interaction with products and technology in both home and work environments (1). In the medical field, it tests actual medical device use by representative users to identify risks in humans' interface with the device. This differs from market research that collects data on opinions and attitudes about the possibility of purchasing the device. The FDA has been increasingly asking for human factor studies before a device is placed on the market in users' hands (1). A sentinel event demonstrating the importance of human factors analysis was the release of a bedside infusion pump in which the "On/Off" switch was located too close to the "Start" key, and nurses were turning the pump off when they intended to start infusions (2). From this point of view one can think of several aspects of human factors that might affect the patients use and adherence to diabetes technology, for example, the technological aspect (e.g., if the pump has a touch screen that is "userfriendly"), the physical contact of devices with the subject (e.g., ease of insertion, ease of wear, and tape issues with sensors and infusion sets), the perceived accuracy of the devices, the effect of knowledge gained from the devices on human interactions (ability of parents to monitor their children and adolescents), and whether these devices increase or decrease the perceived burden of taking care of diabetes. As an example, current CGM devices are not labeled to replace meter glucose readings when making management decisions such as giving insulin or treating hypoglycemia; however, if the user perceives that the sensors are accurate, they could begin making insulin dose decisions based on the sensor and not meter readings. In the present article, some of the studies that have been published from July 2014 to June 2015 that involve human factors will be reviewed and discussed. We have taken a broad view of human factors research to include a number of articles dealing with the interface of pump and sensor technologies in different populations as well as closed-loop studies. We specifically look at how patients are satisfied with the use of a device and whether it is perceived convenient or inconvenient. For example, is the device perceived as userfriendly (easy to learn and operate, comfortable, and does not cause many hassles)? Second, there is the issue of effectiveness of the technology as it relates to their day-today diabetes management; that is, there is an improvement in glycemic control when one diabetes treatment regimen is compared to another.

Those core aspects may have different meanings for different groups. For example, different age groups (toddlers, children, adolescents, young adults, adults, and seniors) can see different advantages and disadvantages in technological devices. The feasibility

\footnotetext{
${ }^{1}$ Jesse Z and Sara Lea Shafer Institute for Endocrinology and Diabetes, National Center for Childhood Diabetes, Schneider Children's Medical Center of Israel, Petah Tikva, Israel.

${ }^{2}$ Stanford Medical Center, Division of Endocrinology and Diabetes, Stanford, CA.
} 
and utility of technological devices also need to fit the environments in which they will be used such as school, the workplace, and/or home. Specific subgroups such as diabetic youth with eating disorders can have unique interactions with diabetes technologies.

In the past year a number of studies concentrated on the interface between technologies such as continuous glucose monitoring, insulin pumps, and artificial pancreas systems and the user. Issues of adherence and motivation as well as user satisfaction are very relevant, and understanding them will help to enhance the use of these technologies. The present article will review articles published in the last year that have studied some of these issues.

\section{Key Articles Reviewed for this Article}

\section{PsychDT Working Group: Report Psychosocial Aspects of Artificial Pancreas} Systems

Barnard $K D^{1}$, Venkat $M V^{2}$, Close $K^{3}$, Heinemann $L^{4}$, Weissberg-Benchall $J^{5}$, Hood $K K^{6}$, Kubiak $T^{7}$, Kowalski $A J^{8}$, Laffel $L^{9}$

J Diabetes Sci Technol 2015; 9: 925-28

Real-time continuous glucose monitoring in type 1 diabetes: a qualitative framework analysis of patient narratives

Pickup JC ${ }^{l}$, Ford Holloway $M^{2}$, Samsi $K^{3}$

Diabetes Care 2015; 38: 544-50

Youth and parent measures of self-efficacy for continuous glucose monitoring: survey psychometric properties

Rasbach $L E^{1,2}$, Volkening $L K^{l}$, Markowitz $J T^{l}$, Butler $D A^{l}$, Katz $M L^{l}$, Laffel $L M^{l}$

Diabetes Technol Ther 2015; 17: 327-34

Salient characteristics of youth with type 1 diabetes initiating continuous glucose monitoring

Telo $G H^{1-3}$, Volkening $L K^{1}$, Butler $D A^{1}$, Laffel $L M^{1}$

Diabetes Technol Ther 2015; 17: 373-78

A remote monitoring system for artificial pancreas support is safe, reliable, and user friendly

Oron $T^{l}$, Farfel $A^{l}$, Muller $I^{I}$, Miller $S^{I}$, Atlas $E^{l}$, Nimri $R^{l}$, Phillip $M^{1,2}$

Diabetes Technol Ther 2014; 16: 699-705

Artificial pancreas (AP) clinical trial participants' acceptance of future AP technology

Bevier $W C^{1}$, Fuller $S M^{2}$, Fuller $R P^{3}$, Rubin $R R^{4}$, Dassau $E^{1,5,6}$, Doyle $F J 3 r d^{1,5,6}$, Jovanovič $L^{1,5,7}$, Zisser $H^{1,5}$

Diabetes Technol Ther 2014; 16: 590-95

Future artificial pancreas technology for type 1 diabetes: what do users want?

Barnard $K D^{1}$, Pinsker $J E^{2}$, Oliver $N^{3}$, Astle $A^{l}$, Dassau $E^{2,4}$, Kerr $D^{2}$

Diabetes Technol Ther 2015; 17: 311-15

Insulin pump-associated adverse events in children and adolescents-a prospective study

Wheeler $B J^{1,2}$, Heels $K^{1}$, Donaghue $K C^{1,3}$, Reith $D M^{2}$, Ambler $G R^{1,3}$

Diabetes Technol Ther 2014; 16: 558-62

Insulin pump use and glycemic control in adolescents with type 1 diabetes: predictors of change in method of insulin delivery across two years

Wong JC ${ }^{1}$, Dolan $L M^{2,3}$, Yang $T T^{4}$, Hood KK

Pediatr Diabetes 2014. [Epub ahead of print] DOI: 10.1111/pedi.12221 


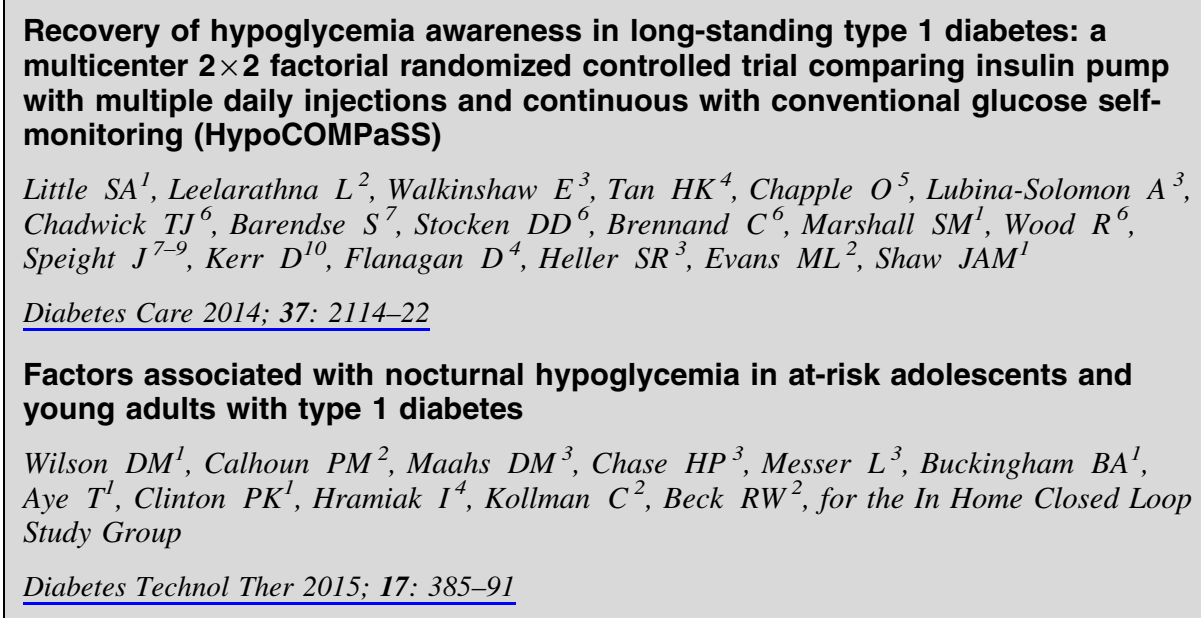

\section{PsychDT Working Group: Report Psychosocial Aspects of Artificial Pancreas Systems}

Barnard $K D^{1}$, Venkat $M V^{2}$, Close $K^{3}$, Heinemann $L^{4}$, Weissberg-Benchall $J^{5}$, Hood KK ${ }^{6}$, Kubiak $T^{7}$, Kowalski $A^{8}{ }^{8}$, Laffel $L^{9}$

${ }^{1}$ Human Development and Health Academic Unit, Faculty of Medicine, University of Southampton, Southampton, UK; ${ }^{2}$ Close Concerns, San Francisco, CA; ${ }^{3}$ The diaTribe Foundation, San Francisco, CA; ${ }^{4}$ Science \& Co, Dusseldorf, Germany; ${ }^{5}$ Northwestern University Feinberg School of Medicine, Ann and Robert H. Lurie Children's Hospital of Chicago, Chicago, IL; ${ }^{6}$ Pediatrics, Psychiatry and Behavioural Sciences, Stanford University School of Medicine, Stanford, CA; ${ }^{7}$ Health Psychology, Johannes Gutenberg University, Mainz, Germany; ${ }^{8}$ Juvenile Diabetes Research Foundation Ltd., New York, NY; ${ }^{9}$ Joslin Diabetes Center, Harvard Medical School, Boston, MA

\section{J Diabetes Sci Technol 2015; 9: 925-28}

\section{Background}

Advances in diabetes technology over recent years have played a central role in diabetes health-care progress. Psychosocial interventions have been shown to have a positive impact on glycemic control, reduce psychological distress, and reduce costs of health-care. Addressing and improving psychosocial outcomes that complement biomedical improvements are crucial to enhance patient acceptance of artificial pancreas (AP) systems.

\section{Methods}

A working group was created to improve collaboration and comparability across different AP research trials.

\section{Results}

Current techniques do not fully reflect the extent to which human and psychological factors play a role in the uptake and efficient use of AP systems. Reliable measures of the psychosocial impact of AP systems for users are crucial to ensure that (i) regulatory authorities are able to robustly consider these aspects as part of their approval process, (ii) govern- ment and private payers are able to factor these aspects into their decisions regarding reimbursement, and (iii) persons with diabetes maximize benefits in terms of both glycemic control and quality of life to minimize the burden of diabetes in everyday life.

\section{Conclusions}

This working group will serve as a platform to foster exchange, identify research needs, and guide and initiate collaborative research laying the groundwork for optimal utilization of diabetes technology in clinical diabetes care. A strong collaboration of all key stakeholders is crucial to ensure that devices are designed, trialed, approved, and provided with minimal user burden and maximum benefit.

\section{Comment}

At the ATTD meeting in February 2015, the first workshop on psychosocial aspects of diabetes technology was held, with an emphasis on artificial pancreas systems. There were over 300 attendees, including clinicians, patients, researchers, industry, and diabetes-focused nonprofit foundations.

Theme 1: Lessons Learned from CGM. Rather than faulting patients for suboptimal adherence, participants suggested that the onus is on the developers of new technologies to create products that patients will want to use (like with other electronic products). Improved accuracy and fewer false alarms have made CGM more attractive to users, and factor calibration and a replacement claim will further enhance the user interface. Psychological measurement instruments can assist in assessments of diabetes technologies with research showing self-efficacy as a key indicator of adherence, motivation, health behavior change, and glycemic outcomes.

Theme 2: A Stepwise Path to the AP. An emerging theme during the discussion was that small step toward AP systems rather than a (quantum) leap to full automation may be the most user-friendly path toward AP systems. While there are already fully automated single or dual hormonal closed-loop systems nearing pivotal 
clinical trials, a stepwise approach could potentially help build trust in the technology by the persons with diabetes (PWD), family members of PWD, and the health-care community as well. It may be that the term "artificial pancreas" does not represent a single technology but rather a series of systems on the path toward full automation. Enabling users to turn on features selectively to provide for a more gradual initiation process may be helpful.

Theme 3: Managing Expectations. Despite enormous enthusiasm for the AP in the scientific and medical communities, many attendees underscored the importance of setting realistic expectations for PWD about what the technology can and cannot do.

Theme 4: The Need for Better Ways to Assess Psychosocial Factors. The discussion touched on the primary goal of the PsychDT working group, which is the development of novel validated tools to assess the psychosocial aspects of diabetes technologies. There was a call for a multicultural approach with new measures ultimately needing to be socially and culturally relevant across a range of settings.

\section{Real-time continuous glucose monitoring in type 1 diabetes: a qualitative framework analysis of patient narratives}

Pickup JC', Ford Holloway $M^{2}$, Samsi $K^{3}$

${ }^{1}$ Diabetes Research Group, King's College London School of Medicine, Guy's Hospital, London, UK; ${ }^{2}$ INPUT Patient Advocacy, London, UK; ${ }^{3}$ Social Care Workforce Research Unit, School of Social Science and Public Policy, King's College London, London, UK

Diabetes Care 2015; 38: 544-50

\section{Objective}

In this study the narratives of people with type 1 diabetes concerning their experiences with real-time continuous glucose monitoring (CGM) were analyzed.

\section{Research Design and Methods}

Subjects with type 1 diabetes using CGM and their caregivers filled an online questionnaire. The questions included duration of CGM, sensor wear frequency, funding, and a free narrative about experiences or views about CGM. The authors used qualitative framework analysis to analyze 100 responses; $50 \%$ of participants were 18 years old or younger.

\section{Results}

Most participants (87\%) used CGM with pumps, 71\% used sensors $\geq 75 \%$ of the time, and $66 \%$ received funding for CGM from the National Health Service. The authors identified four main themes: (i) metabolic control, (ii) living with CGM (work and school, sleep, exercise, nutrition, frequency of self-monitoring of blood glucose), (iii) psychological issues and patient/caregiver attitudes, and (iv) barriers to CGM use (technical issues, financial issues, attitudes of health-care professionals toward CGM). Although some hassles were reported, experiences were overwhelmingly positive, with improvement in glycemic control, diet and exercise management, quality of life, and physical and psychological well-being, as well as reduced frequency of SMBG. Technical problems included sensor inaccuracy and unreliability, and "alarm fatigue." It is noted that several subjects reported of the advantage in using CGM with an insulin pump with automatic suspension of insulin delivery during hypoglycemia that helped in reducing hypoglycemia frequency and fear of nocturnal hypoglycemia.

\section{Conclusions}

The narratives of both patients and caregivers indicate that for many of them CGM is a valuable supplement to their management of diabetes.

\section{Comment}

In recent years, several studies have investigated the subjective experience and attitudes of patients with type 1 diabetes concerning the use of continuous glucose monitors (CGM).

Most of these studies utilized self-reported Likert scale questionnaires $(1,2)$. For example, in the continuous glucose monitoring satisfaction scale the participant is asked to rate sentences about the possible hassles and benefits of CGM use on a scale from 1 to 5 (e.g., "allows more freedom in daily life" or "has been harder or more complicated than expected"').

The current study takes this methodology one step further by using a qualitative framework of patients' narratives about their experiences with real-time continuous glucose monitoring. This methodological technique enables us to understand in more subtle ways the nuances of continuous glucose monitoring use from the subjects' point of view.

There were many negative comments about alarms being annoying, the intrusion of alarms, disturbed sleep, and a life "living by alarms." Most believed that their sensors were "not accurate or reliable enough." This may be because $70 \%$ of the CGM users were on a Medtronic sensor, and only $10 \%$ were using a Dexcom G4P sensor. Sensor use in the United States has increased significantly over the last several years, predominately based on a perceived increase accuracy of the Dexcom G4P sensor, and a lower false alarm rate. As newer generations of the Medtronic sensor are introduced, many of the perceived sensor inaccuracy issues should resolve.

It was interesting to see that, for a parent of a 4-yearold girl, the experience of CGM use was positive because there were "less variations of blood glucose levels" despite the fact that "the HbA1c level has dropped only a little," or "being able to see exactly what impact different foods have on my blood sugar over several hours." The narrative framework analysis provides more direct information about the patients' experience using the CGM. 


\section{Youth and parent measures of self-efficacy for continuous glucose monitoring: survey psychometric properties}

Rasbach $L E^{1,2}$, Volkening $L K^{l}$, Markowitz $J T^{l}$, Butler DA ${ }^{1}$, Katz $M L^{1}$, Laffel $L M^{1}$

${ }^{1}$ Pediatric, Adolescent, and Young Adult and Genetics and Epidemiology Sections, Joslin Diabetes Center, Harvard Medical School, Boston, MA; ${ }^{2}$ Medical University of South Carolina College of Nursing, Charleston, SC

Diabetes Technol Ther 2015; 17: 327-34

This manuscript is discussed also in the article on: Diabetes Technology and Therapy in the Pediatric Age Group, p. S-91.

\section{Background}

This study aimed to assess the predictive validity of a novel CGM Self-Efficacy (CGM-SE) survey on CGM use and hemoglobin A1c (HbA1c) levels.

\section{Subjects and Methods}

Sixty-one youths in the control arm of a 2-year study on CGM use were enrolled in this study. Youth and parents completed the CGM-SE surveys at randomization after a 1week run-in to assess CGM tolerability. Analyses of predictive validity were based on CGM use and $\mathrm{HbA1c}$ outcomes after 3 and 6 months of CGM use.

\section{Results}

At study entry, youth were $12.7 \pm 2.7$ years old with diabetes duration of $6.1 \pm 3.6$ years and an HbA1c level of $8.0 \pm 0.8 \%$ $(64 \pm 9 \mathrm{mmol} / \mathrm{mol})$, blood glucose monitoring frequency was $6.8 \pm 2.4$ times/day, and $84 \%$ received pump therapy. CGMSE surveys had acceptable internal consistency (Cronbach's $\alpha=0.80$ for youth and 0.82 for parents). Youth reporting higher baseline CGM self-efficacy (CGM-SE score of >80) had significantly greater CGM use and lower HbA1c level after 3 and 6 months compared with youth reporting lower baseline CGM self-efficacy (CGM-SE score of $\leq 80$ ).

\section{Conclusions}

The CGM-SE surveys appear to have strong psychometric properties. CGM self-efficacy may offer an opportunity to assess the likelihood of CGM adherence and glycemic improvement in youth with type 1 diabetes in clinical and research settings.

\section{Comment}

Although consistent use of continuous glucose monitoring among children and adolescents can improve significantly their glycemic control, it is well known that this age group demonstrates poor adherence as well as lack of motivation in their diabetes self-care.

The concept of self-efficacy is defined as "the belief that one can carry out specific behaviors in specified situations" (3). Previous studies have shown that better sense of self-efficacy among adolescents with type 1 diabetes may empower their adherence and motivation and can be an important indicator of health behavior change (4).
The current study suggests that the psychological concept of self-efficacy can be applied specifically to adolescents' attitude toward continuous glucose monitoring and can serve as a framework for developing a psychometric measure of CGM challenges (i.e., technical issues, parent-child problems, or user-friendly issues such as false alarms). This questionnaire can help the diabetes multidisciplinary team to trace adolescent patients with low self-efficacy and to improve their adherence and motivation for CGM use.

\section{Salient characteristics of youth with type 1 diabetes initiating continuous glucose monitoring}

Telo $\mathrm{GH}^{1-3}$, Volkening $\mathrm{LK}^{1}$, Butler $D A^{1}$, Laffel $L M^{1}$

${ }^{1}$ Pediatric, Adolescent, \& Young Adult and Genetics and Epidemiology Sections, Joslin Diabetes Center, Harvard Medical School, Boston, MA; ${ }^{2}$ Universidade Federal do Rio Grande do Sul, Porto Alegre, Brazil; ${ }^{3}$ CAPES Foundation, Ministry of Education, Brasilia, Brazil

Diabetes Technol Ther 2015; 17: 373-78

\section{Objective}

Youth with type 1 diabetes find CGM use challenging. This study aimed to investigate patient and family behavioral and clinical characteristics associated with implementing CGM.

\section{Research Design and Methods}

The study compared 120 youth interested in starting CGM (the CGM group) with a general sample of 238 youth with type 1 diabetes (the standard group). In a cross-sectional study, youth and their parents completed validated surveys assessing adherence to diabetes management, diabetes-specific family conflict, parent involvement in diabetes management, and youth quality of life. Demographic and clinical data were obtained from chart review and interview.

\section{Results}

Youth participants had a mean age of $13.0 \pm 2.8$ years, diabetes duration of $6.3 \pm 3.4$ years, and hemoglobin A1c (HbAlc) level of $8.2 \pm 1.0 \%(66 \pm 11 \mathrm{mmol} / \mathrm{mol})$. Youth in the CGM group performed more frequent blood glucose monitoring, had lower HbA1c levels, and were more likely to be treated by continuous subcutaneous insulin infusion (CSII) and to be living in two-parent homes than youth in the standard group. Compared with the standard group, youth interested in wearing a CGM device and their parents reported greater adherence to diabetes management, less diabetes-specific family conflict, and higher youth quality of life. No differences were found between groups with respect to parent involvement in diabetes management.

\section{Conclusions}

In efforts to enhance CGM uptake, it is important to address factors such as blood glucose monitoring frequency, CSII use, adherence, and diabetes-specific family conflict when considering youth with type 1 diabetes for CGM. 


\section{Comment}

Diabetes care in children and adolescents cannot rely solely on adolescent self-care. Several empirical studies in the recent years have shown that the involvement of parents in their children's diabetes management will help them to be more adherent, make less mistakes in their self-care, and will have better metabolic control than youth whose parents are less involved and engaged in diabetes tasks at home $(5,6)$.

On the other hand, the complexity and demanding nature of diabetes regimen may have a profound impact on many fundamental aspects of daily family life and in turn might increase the stress and conflict in the family. Many empirical studies have documented that higher levels of diabetes-specific conflict in the family are associated with lower levels of child adherence to the diabetes management regimen (7).

One of the main reasons for the development of the diabetes-specific family conflict is that the adolescent is going through the developmental phase known as the "second separation-individuation" (8); in this phase the adolescent must disengage from his parents and establish a sense of self that is distinct and individuated. On the other hand, many adolescents do not have the mature abilities of taking full responsibility over their diabetes management and lack a future perspective of the consequences of their poor adherence. This in turn forces the parents to be more involved in the diabetes care and increases the conflict.

The diabetes-specific family conflict may be even higher when using demanding technologies such as continuous glucose monitoring. It is well known that alongside the benefits of CGM use, many patients report about hassles and technical difficulties.

The diabetes multidisciplinary team must be aware of the role of parents in the adolescents' diabetes management and to the potential and the risk in such involvement. This article again demonstrates that the strongest predictor of interest in CGM use is a person's interest in managing their diabetes. In the JDRF randomized clinical trial after factoring out age, the only predictor of using a CGM for 6 or more days a week was the selfreported frequency of fingerstick glucose readings before beginning CGM use (9). Sex, race, pump use, baseline A1c, recent severe hypoglycemia, parental education level, and household income did not predict future CGM use in this study even when CGM devices were provided to the subjects.

\section{A remote monitoring system for artificial pancreas support is safe, reliable, and user friendly}

Oron $T^{l}$, Farfel $A^{l}$, Muller $I^{1}$, Miller $S^{l}$, Atlas $E^{l}$, Nimri $R^{l}$, Phillip $M^{1,2}$

${ }^{1}$ Jesse $Z$ and Sara Lea Shafer Institute for Endocrinology and Diabetes, National Center for Childhood Diabetes, Schneider Children's Medical Center of Israel, Petah-Tikva, Israel; ${ }^{2}$ Sackler Faculty of Medicine, Tel Aviv University, Tel Aviv, Israel

Diabetes Technol Ther 2014; 16: 699-705
This manuscript is also discussed in the article on: Diabetes Technology and Therapy in the Pediatric Age Group, p. S-93.

\section{Introduction}

A remote monitoring and control diabetes management system (MDRS) supporting the at-home use of an artificial pancreas (AP) was developed by the authors of this study, which aimed at assessing the efficacy and safety of the MDRS. In addition, the study aimed at assessing this unique system's ability to prevent or shorten nocturnal hypoglycemia episodes without the use of an AP and evaluated parental attitudes about using this type of system in the future.

\section{Materials and Methods}

This was a prospective, case-control, randomized study. Eighteen patients treated nightly over a 2 -week period by the MDRS were compared with 19 patients treated with sensoraugmented pump (SAP) therapy. They analyzed the performance of the MDRS, the number and duration of hypo- or hyperglycemia episodes, and the efficacy of the MDRS in detecting them. Parents of the study participants completed the hypoglycemia fear survey, attitude, and satisfaction questionnaires.

\section{Results}

The MDRS allows continuous monitoring of the patients using it. Without the use of an AP, the MDRS did not significantly prevent nocturnal hypoglycemia episodes. The patients and their parents found the system reliable and userfriendly. The overall impression of the MDRS users was favorable, with a keen interest of the patients and their caregivers to use such a system in the future.

\section{Conclusions}

For at-home AP systems, the MDRS is reliable and safe. Remote monitoring of type 1 diabetes patients treated with SAP therapy is a promising and feasible task that is highly anticipated by patients and their caregivers.

\section{Artificial pancreas (AP) clinical trial participants' acceptance of future AP technology}

Bevier $W C^{1}$, Fuller $S M^{2}$, Fuller $R P^{3}$, Rubin $R R^{4}$, Dassau $E^{1,5,6}$, Doyle FJ $3 r^{1,5,6}$, Jovanovič $L^{1,5,7}$, Zisser $H^{1,5}$

${ }^{1}$ Sansum Diabetes Research Institute, Santa Barbara, CA; ${ }^{2}$ Department of Family and Consumer Sciences, University of Arkansas Division of Agriculture Research and Extension, Little Rock, AR; ${ }^{3}$ Department of Speech Communication, University of Arkansas at Little Rock, Little Rock, AR; ${ }^{4}$ Departments of Medicine and Pediatrics, Johns Hopkins University School of Medicine, Baltimore, MD; ${ }^{5}$ Department of Chemical Engineering, University of California Santa Barbara, Santa Barbara, CA; ${ }^{6}$ Institute for Collaborative Biotechnologies, University of California Santa Barbara, Santa Barbara, CA; ${ }^{7}$ Biomolecular Science \& Engineering Program, University of California Santa Barbara, Santa Barbara, CA

Diabetes Technol Ther 2014; 16: 590-95 


\section{Background}

AP systems are an active field of diabetes research. The attitudes of AP clinical trial participants toward acceptance of the technology were examined in this pilot study.

\section{Subjects and Methods}

After possible influencers of AP technology adoption were considered, a survey with 34 questions was developed. The questionnaire assessed current treatment satisfaction, dimensions of clinical trial participant motivation, and variables of the technology acceptance model (TAM). Forty-seven subjects were contacted to complete the survey. The reliability of the survey scales was tested using Cronbach's $\alpha$. Using regression analysis, the study explored the relationship of the factors to the likelihood of AP technology adoption.

\section{Results}

Thirty-six subjects (76.6\%) completed the survey. Of the respondents, $86.1 \%$ were either highly likely or likely to adopt the technology once available. Reliability analysis of the survey dimensions revealed good internal consistency, with scores of $>0.7$ for current treatment satisfaction, convenience (motivation), personal health benefit (motivation), perceived ease of use (TAM), and perceived usefulness (TAM). Linear modeling showed that future acceptance of the AP was significantly associated with TAM and the motivation variables of convenience plus the individual item benefit to others $(R(2)=0.26, p=0.05)$. When insulin pump and continuous glucose monitor use were added, the model significance improved $(R(2)=0.37, p=0.02)$.

\section{Conclusions}

Individuals with direct AP technology experience expressed high likelihood of future acceptance. Results support the factors of personal benefit, convenience, perceived usefulness, and perceived ease of use as reliable scales that suggest system adoption in this highly motivated patient population.

\section{Comment}

In these two studies, participants and their caregivers report their attitude toward future technology. For example, in the first study there were significant differences between parental attitudes toward sensor augmented pump therapy (i.e., the accuracy of the sensor) and the remote monitoring system (monitoring and control diabetes management system, MDRS). Seventyfour percent of subjects stated that the remote monitoring system fulfilled their expectations. However, only 52\% felt that the sensor was accurate or reliable, and $34 \%$ of the parents completely shut off the alarms at night so that their sleep would not be interrupted. The main concern of the system was accuracy and reliability of the sensor, but they would be very interested in using remote monitoring, and approximately $90 \%$ of the parents reported that they were willing to be connected to a remote monitoring system and that they would feel more confident in regard to nocturnal hypoglycemia episodes with remote nighttime monitoring. The sensor is critical for remote monitoring to function. In an earlier study using a
Dexcom G4P sensor (10), 79\% of sensor-detected hypoglycemic events were true, and remote monitoring significantly decreased hypoglycemic events and the duration of hypoglycemia.

The second study examined the attitudes of participants who had participated in clinical artificial pancreas trials toward future acceptance of artificial pancreas systems. Subjects expressed a high likelihood of future acceptance of artificial pancreas systems. These studies collected information from participants after they had completed research studies and use a very important technique in human factor research, namely, the "walkthrough" technique. During the walk-through, participants are questioned and encouraged to provide feedback on difficulties they notice while using the device. Evaluators can also collect subjective information from participants about their mental processes, physical interactions, and perceived workloads (intellectual, physical, and emotional) when using the systems (11).

Integrating the feedback of people with diabetes and their caregivers can not only improve the product but can also help to understand the psychological aspects of using these systems and their influence on treatment satisfaction and quality of life.

\section{Future artificial pancreas technology for type 1 diabetes: what do users want?}

Barnard $K D^{1}$, Pinsker $J E^{2}$, Oliver $N^{3}$, Astle $A^{1}$, Dassau $E^{2,4}$, Kerr $D^{2}$

${ }^{1}$ Human Development and Health, Faculty of Medicine, University of Southampton, Southampton, UK; ${ }^{2}$ William Sansum Diabetes Center, Santa Barbara, CA; ${ }^{3}$ Imperial College London, London, UK; ${ }^{4}$ Department of Chemical Engineering, University of California Santa Barbara, Santa Barbara, $C A$

Diabetes Technol Ther 2015; 17: 311-15

\section{Background}

Technical achievements, such as time in range for glucose levels or prevention of hypoglycemia, have been the primary focus of AP research. Few studies have aimed to determine the expectations of users of AP technology.

\section{Subjects and Methods}

Persons with type 1 diabetes and parents of children with type 1 diabetes were invited to take part in an online survey concerning future use and expectations of AP technology. The survey was advertised via Twitter, Facebook, and DiabetesMine, plus advocacy groups and charities, including INPUT, Diabetes UK, and the Diabetes Research and Wellness Foundation. Quantitative responses were categorized on a five-point Likert scale. Using content analysis, free text responses were examined.

\section{Results}

Over the period of a month, 266 surveys were completed. In all, 240 participants indicated that they were highly likely 
to use a fully automated 24-hour AP. Approximately half of the respondents indicated that they would be likely to use a device that only functioned overnight. The top reasons for not wanting an AP included size, visibility, and lack of effectiveness. Despite perceived potential downsides, participants indicated a strong need for a device that will help minimize the burden of disease and help improve psychosocial functioning and quality of life.

\section{Conclusions}

Alongside biomedical and engineering excellence, the views of people who would use an AP are crucial in the development of such devices to ensure that they are fit for use. Without this, it is unlikely that an AP will be sufficiently successful to meet the needs of users and to achieve their ultimate goals.

\section{Comment}

In this online survey, participants were asked to express their expectations about the future design. The survey question presented to the participants, "What would a successful artificial pancreas look like for you?" allows the artificial pancreas development team to have a better understanding of what the patient considers important. Certain parameters might match the team expectations (i.e., size, effective, no canula) but some might be surprising and give some food for thought that will facilitate developing a better device that will enhance the users' satisfaction.

\section{Insulin pump-associated adverse events in children and adolescents-a prospective study}

Wheeler BJ ${ }^{1,2}$, Heels $K^{1}$, Donaghue $K C^{1,3}$, Reith $\mathrm{DM}^{2}$, Ambler $G R^{1,3}$

${ }^{1}$ The Institute of Endocrinology and Diabetes, The Children's Hospital at Westmead, Sydney, Australia; ${ }^{2}$ Department of Women's and Children's Health, University of Otago, Dunedin, New Zealand; ${ }^{3}$ Discipline of Paediatrics and Child Health, The University of Sydney, Sydney, Australia

\section{Diabetes Technol Ther 2014; 16: 558-62}

\section{Background}

Insulin pumps (CSII) are frequently used to achieve intensive insulin therapy in the management of type 1 diabetes mellitus. Although there have been many reports on CSII outcomes, there is little recent data on CSII-associated adverse events (AEs) and their incidence and characteristics.

\section{Subjects and Methods}

Phone calls to their 24-hour diabetes support service were screened for CSII-associated AEs. Within 96 hours of an event, phone interviews were conducted with the parent/ patient. These interviews examined $\mathrm{AE}$ characteristics and the role of the user, as well as questions relating to outcome and the impact to the family and patient. Comparisons were made with clinic CSII patients not reporting an AE.

\section{Results}

In all, 50 confirmed AEs occurred in 45 of 405 (11.1\%) patients over a 16-week study period. This was annualized to an AE incidence of 40 AEs/100 person-years. Pump malfunction and infusion set/site failures were the most common events reported, occurring in $27(54.0 \%)$ and $18(36.0 \%)$ AEs, respectively. A user- or education-related issue was implicated in $22(44.0 \%)$ events. Pump replacement occurred in 19 of 50 occurrences $(38.0 \%)$. Additionally, $16(32.0 \%)$ reported a hospital admission or emergency department attendance as a consequence. When compared with those on CSII not reporting an AE, AEs were associated with age $<10$ years (odds ratio $=3.2[95 \%$ confidence interval, 1.7-6.1]) but not with gender, glycosylated hemoglobin, diabetes duration, or pumping duration.

\section{Conclusions}

This is the first prospective study to look at AEs in modern insulin pumps. AEs appear to be a common occurrence and should be anticipated. Their origin is multifactorial, with the pump, associated consumables, and the user all being important factors. To minimize pump-associated AEs and their impact, ongoing support and anticipatory education are crucial.

\section{Comment}

This study is the second of two studies examining the phenomenon of CSII adverse events in the child and adolescent population and its relation to subjective measures such as family perceptions of psychosocial and educational issues (the first study from the same research group was retrospective) (12).

Previous studies have mentioned several types of pump adverse events as a reason for CSII discontinuation. For example, De Vries et al. found that technical problems (e.g., recurrent catheter obstruction, technical fault, and infusion site difficulty) occurred in $25 \%$ of the patients. The proportion of psychological issues as a cause for pump discontinuation was significantly higher (31\%) (13).

Other studies have shown that the most commonly stated reasons for stopping use of CSII therapy related mainly to catheter and sociopsychological factors (such as visibility and the pump feeling like a foreign body) (14).

In the current study, insulin pump adverse events are viewed in a wider perspective: not just the narrow technical aspects of CSII use, but also the psychosocial aspects. Pump failures caused significant parent anxiety/ worry in $70 \%$ of failures, and $52 \%$ of the time caused a significant impact on the family schedule, and $34 \%$ of the time required time of work. In children, $58 \%$ of these events caused significant anxiety/worry and $42 \%$ of events required that they take time off from school.

\section{Insulin pump use and glycemic control in adolescents with type 1 diabetes: predictors of change in method of insulin delivery across two years}

Wong $J C^{1}$, Dolan $L M^{2,3}$, Yang $T T^{4}$, Hood $K K^{1}$ 
${ }^{1}$ Division of Endocrinology, Department of Pediatrics, University of California San Francisco, San Francisco, CA; ${ }^{2}$ Department of Pediatrics, University of Cincinnati College of Medicine, Cincinnati, OH; ${ }^{3}$ Division of Endocrinology, Cincinnati Children's Hospital Medical Center, Cincinnati, $\mathrm{OH} ;{ }^{4}$ Division of Child and Adolescent Psychiatry, Department of Psychiatry, University of California San Francisco, San Francisco, CA

Pediatr Diabetes 2014. [Epub ahead of print] DOI: 10.1111/ pedi.12221

\section{Background}

Very few studies have looked at the durability of CSII use, and none have explored the connection between depression and CSII discontinuation.

\section{Subjects and Methods}

The authors used mixed models with data from 150 youth with type 1 diabetes visiting every 6 months over 2 years to explore the connection between depressive symptoms (measured by the Children's Depression Inventory [CDI]), method of insulin regimen, and hemoglobin A1c,

\section{Results}

Of the $63 \%$ who used CSII, compared with multiple daily injections (MDI) at baseline, there were higher proportions who were nonminorities, had caregivers with a college degree, had private insurance, and had two caregivers in the home $(p \leq 0.01)$. After adjusting for time, sex, age, T1D duration, frequency of blood glucose monitoring, ethnicity, insurance, and caregiver number and education, baseline pump use was associated with $-0.79 \%$ lower mean A1c (95\% confidence interval $[\mathrm{CI}]:-1.48,-0.096 ; p=0.03$ ). For participants who used pumps at baseline but switched to MDI during the study $(n=9)$, the mean A1c was $1.38 \%$ higher (95\% CI: $0.68,2.08 ; p<0.001)$ compared to those who did not switch. A 10-point increase in CDI was associated with a $0.39 \%$ increase in A1c (95\% CI: $0.16,0.61 ; p=0.001)$, independent of pump use. Concerning the temporal relationship between CDI score and changing method of insulin delivery, prior higher CDI scores were associated with changing from CSII to MDI (odds ratio $=1.21 ; 95 \%$ CI: 1.05 , $1.39 ; p=0.007)$. Clinicians should recognize the correlations between depressive symptoms, change in insulin delivery method, and the influence on glycemic control.

\section{Comment}

Diabetes treatment regimens create a high level of demands on the patient. Many adolescents with type 1 diabetes report that the unceasing burden of diabetes management causes burnout, psychological distress, and family conflicts and in many cases even depression. In fact, depression is considered to be the most common psychiatric diagnosis of adolescents with type 1 diabetes (15).

Findings indicate that approximately one in seven adolescents with diabetes met the clinical criteria for depression according to their self-report. This level of depressive symptoms in children and adolescents with type 1 diabetes is nearly twice as high as in general population (16). Depressive symptoms may lead to poor adherence to diabetes treatment with less frequent blood glucose monitoring and missed insulin boluses and in some cases may even lead to insulin pump discontinuation.

The current study suggests that depressive symptoms may lead to a change in attitudes or behaviors around diabetes, such as CSII dissatisfaction, or feeling that the pump is not working well or is too burdensome. The diabetes team should be aware that complaints about technical difficulties or about attitudes toward the insulin pump may reflect the patients' psychological state.

\section{Recovery of hypoglycemia awareness in long-standing type 1 diabetes: a multicenter $2 \times 2$ factorial randomized controlled trial comparing insulin pump with multiple daily injections and continuous with conventional glucose self-monitoring (HypoCOMPaSS)}

Little $S A^{1}$, Leelarathna $L^{2}$, Walkinshaw $E^{3}$, Tan $H K^{4}$, Chapple $O^{5}$, Lubina-Solomon $A^{3}$, Chadwick $T J^{6}$, Barendse $S^{7}$, Stocken $D D^{6}$, Brennand $C^{6}$, Marshall $S M^{1}$, Wood $R^{6}$, Speight $J^{7-9}$, Kerr $D^{10}$, Flanagan $D^{4}$, Heller $S R^{3}$, Evans $M L^{2}$, Shaw JAM ${ }^{1}$

${ }^{1}$ Institute of Cellular Medicine, Newcastle University, Newcastle, UK; ${ }^{2}$ Wellcome Trust-MRC Institute of Metabolic Science, Metabolic Research Laboratories, University of Cambridge, Cambridge, UK; ${ }^{3}$ School of Medicine and Biomedical Sciences, Sheffield University, UK; ${ }^{4}$ Peninsula College of Medicine and Dentistry, Plymouth, UK; ${ }^{5}$ Bournemouth Diabetes and Endocrine Centre, Royal Bournemouth Hospital, Bournemouth, UK, ${ }^{6}$ Newcastle Clinical Trials Unit, Institute of Health and Society, Newcastle University, Newcastle, UK; ${ }^{7}$ AHP Research, Hornchurch, UK; ${ }^{8}$ The Australian Centre for Behavioural Research in Diabetes, Diabetes Australia-Vic, Melbourne, Australia; ${ }^{9}$ Centre for Mental Health and Wellbeing Research, School of Psychology, Deakin University, Burwood, Australia; ${ }^{10}$ Centre for Postgraduate Medical Research and Education, Bournemouth University, UK

Diabetes Care 2014; 37: 2114-22

This manuscript is also discussed in the article on: Insulin Pumps, p. S-27.

\section{Objective}

In order to assess whether impaired awareness of hypoglycemia (IAH) can be improved and severe hypoglycemia (SH) can be prevented in type 1 diabetes, the authors conducted a study for comparing between an insulin pump (CSII) with multiple daily injections (MDI) and real-time continuous glucose monitoring (RT) with conventional selfmonitoring of blood glucose (SMBG).

\section{Research Design and Methods}

Adults with type 1 diabetes and IHA participated in a 24 -week $2 \times 2$ factorial randomized controlled trial. All participants received similar education, support, and congruent therapeutic targets aimed at rigorous avoidance of biochemical 
hypoglycemia without relaxing overall control. Primary end point was between-intervention difference in 24-week hypoglycemia awareness (Gold score).

\section{Results}

Ninety-six subjects (mean diabetes duration 29 years) were randomized. Overall, biochemical hypoglycemia $(\leq 3.0 \mathrm{mmol} / \mathrm{L})$ declined $(53 \pm 63$ to $24 \pm 56 \mathrm{~min} / 24 \mathrm{~h} ; p=0.004$ [ $t$ test $])$ without deterioration in $\mathrm{HbA1c}$. Hypoglycemia awareness improved $(5.1 \pm 1.1$ to $4.1 \pm 1.6 ; p=0.0001$ [ $t$ test] $)$ with decreased SH (8.9 \pm 13.4 to $0.8 \pm 1.8$ episodes/patient-year; $p=0.0001[t$ test $])$. At 24 weeks, there was no significant difference in awareness comparing CSII with MDI (4.1 \pm 1.6 vs. $4.2 \pm 1.7$; difference $0.1 ; 95 \% \mathrm{CI}-0.6$ to 0.8$)$ and RT with SMBG ( $4.3 \pm 1.6$ vs. $4.0 \pm 1.7$; difference $-0.3 ; 95 \%$ CI -1.0 to 0.4 ). Between-group analyses demonstrated comparable reductions in $\mathrm{SH}$, fear of hypoglycemia, and insulin doses with equivalent $\mathrm{HbA1c}$. Treatment satisfaction had better scores with CSII than MDI (32 \pm 3 vs. $29 \pm 6 ; p=0.0003$ [ $t$ test]), but comparable with SMBG and RT (30 55 vs. $30 \pm 5 ; p=0.79[t$ test $])$.

\section{Conclusions}

The authors found out that hypoglycemia awareness can be improved and frequent severe hypoglycemia can be prevented in long-standing type 1 diabetes without relaxing HbA1c. Identical biomedical results were achieved with conventional MDI and SMBG regimens compared with CSII and RT, although satisfaction was better with insulin pump therapy.

\section{Factors associated with nocturnal hypoglycemia in at-risk adolescents and young adults with type 1 diabetes}

Wilson $\mathrm{DM}^{1}$, Calhoun $P M^{2}$, Maahs $D M^{3}$, Chase $H P^{3}$, Messer $L^{3}$, Buckingham $B A^{1}$, Aye $T^{1}$, Clinton $P K^{1}$, Hramiak $I^{4}$, Kollman $C^{2}$, Beck $R W^{2}$, for the In Home Closed Loop Study Group

${ }^{1}$ Stanford University, Stanford, CA; ${ }^{2}$ Jaeb Center for Health Research, Tampa, FL; ${ }^{3}$ Barbara Davis Center for Childhood Diabetes, Aurora, CO; and ${ }^{4}$ St. Joseph's Health Care, London, Ontario, Canada

Diabetes Technol Ther 2015; 17: 385-91

\section{Background}

Nocturnal hypoglycemia is a particularly dangerous impediment to good glycemic control. Understanding the major contributors to nocturnal hypoglycemia remains crucial for understanding and mitigating risk.

\section{Material and Methods}

Continuous glucose monitoring (CGM) data for 855 nights were studied, generated by 45 subjects $15-45$ years of age with hemoglobin A1c (HbA1c) levels of $\leq 8.0 \%$ who participated in a larger randomized study. Factors assessed for potential association with nocturnal hypoglycemia (CGM measurement of $<60 \mathrm{mg} / \mathrm{dL}$ for $\geq 30 \mathrm{~min}$ ) included bedtime blood glucose (BG), exercise intensity, bedtime snack, in- sulin on board, day of the week, previous daytime hypoglycemia, age, gender, HbA1c level, diabetes duration, daily basal insulin, and daily insulin dose.

\section{Results}

Hypoglycemia occurred during 221 of 885 (25\%) nights and was more frequent with the following factors: younger age $(p<0.001)$, lower HbA1c levels $(p=0.006)$, medium/ high-intensity exercise during the preceding day $(p=0.003)$, and the occurrence of antecedent daytime hypoglycemia $(p=0.001)$. There was a trend for lower bedtime BG levels to be associated with more frequent nocturnal hypoglycemia $(p=0.10)$. The following elements were not associated with nocturnal hypoglycemia: bedtime snack, before bedtime insulin bolus, weekend versus weekday, gender, and daily basal and bolus insulin.

\section{Conclusions}

It may help patients and providers decrease the risk of hypoglycemia at night by making them aware that $\mathrm{HbA} 1 \mathrm{c}$ level, exercise, bedtime BG level, and daytime hypoglycemia are all modifiable factors associated with nocturnal hypoglycemia. Risk for nocturnal hypoglycemia increased in a linear fashion across the range of variables, with no clear-cut thresholds to guide clinicians or patients for any particular night.

\section{Comment}

Hypoglycemia is described as one of the most disturbing phenomena in diabetes management. Nocturnal hypoglycemia and hypoglycemia unawareness are the main contributors to the development of fear of hypoglycemia symptoms, including intentional decreases in insulin dosing, resulting in elevated glucose levels and increased HbA1c (17).

Two important conclusions can be drawn from these two studies. The first is that there are modifiable behaviors that can take into account exercise, bedtime BG levels, and daytime hypoglycemia to decrease nocturnal hypoglycemia. The second is that a comprehensive educational program including nutritional aspects (e.g., DAFNE program) and psychological aspects (e.g., cognitive behavioral therapy for fear of hypoglycemia and blood glucose awareness training programs) (18) with either MDI or CSII therapy and frequent self-monitoring of glucose levels with or without CGM therapy can reduce hypoglycemia unawareness.

\section{Author Disclosure Statement}

No conflicts exist for A.L. B.B. is on the medical advisory boards of Medtronic Diabetes, Novo-Nordisk, Tandem, Sanofi, and Convatec. He has received research support from Medtronic Diabetes, Roche and Dexcom.

\section{References}

1. Chase HP, Beck RW, Xing D, Tamborlane WV, Coffey J, Fox LA, Ives B, Keady J, Kollman C, Laffel L, Ruedy KJ. 
Continuous glucose monitoring in youth with type 1 diabetes: 12-month follow-up of the Juvenile Diabetes Research Foundation continuous glucose monitoring randomized trial. Diabetes Technol Ther 2010; 12: 507-15.

2. JDRF Continuous Glucose Monitoring Study Group. Validation of measures of satisfaction with and impact of continuous and conventional glucose monitoring. Diabetes Technol Ther 2010; 12: 679-84.

3. Bandura A. Self-efficacy: toward a unifying theory of behavioral change. Psychol Rev 1977; 84: 191-215.

4. Cardwell MS. Improving medical adherence in women with gestational diabetes through self-efficacy. Clin Diabetes 2013; 31: 110-15.

5. Anderson BJ, Vangsness L, Connell A, Butler D, Goebel Fabbri A, Laffel LM. Family conflict, adherence, and glycaemic control in youth with short duration type 1 diabetes. Diabet Med 2002; 19: 635-42.

6. Wysocki T, Taylor A, Hough BS, Linscheid TR, Yeates KO, Naglieri JA. Deviation from developmentally appropriate self-care autonomy. Association with diabetes outcomes. Diabetes Care 1996; 19: 119-25.

7. Markowitz JT, Pratt K, Aggarwal J, Volkening LK, Laffel LM. Psychosocial correlates of continuous glucose monitoring use in youth and adults with type 1 diabetes and parents of youth. Diabetes Technol Ther 2012; 14: 523-26.

8. Blos P. The Adolescent Passage. Madison, CT: International Universities Press, 1979.

9. Juvenile Diabetes Research Foundation Continuous Glucose Monitoring Study Group. Factors predictive of use and of benefit from continuous glucose monitoring in type 1 diabetes. Diabetes Care 2009; 32: 1947-53.

10. DeSalvo DJ, Keith-Hynes P, Peyser T, Place J, Caswell K, Wilson DM, Harris B, Clinton P, Kovatchev B, Buckingham BA. Remote glucose monitoring in camp setting reduces the risk of prolonged nocturnal hypoglycemia. Diabetes Technol Ther 2014; 16: 1-7.
11. Medical device use-safety: Incorporating human factors engineering into risk management. Guidance for industry and FDA premarket and design control reviewers. Document issued on July 18, 2000 [article online]. www.fda.gov/ downloads/medicaldevices/deviceregulationandguidance/ guidancedocuments/ucm094461.pdf

12. Wheeler BJ, Donaghue KC, Heels K, Ambler GR. Family perceptions of insulin pump adverse events in children and adolescents. Diabetes Technol Ther 2014; 16: 204-7.

13. de Vries L, Grushka Y, Lebenthal Y, Shalitin S, Phillip M. Factors associated with increased risk of insulin pump discontinuation in pediatric patients with type 1 diabetes. Pediatr Diabetes 2011; 12: 506-12.

14. Seereiner $S$, et al. Attitudes towards insulin pump therapy among adolescents and young people. Diabetes Technol Ther 2010; 12: 89-94.

15. Lawrence JM, Standiford DA, Loots B, Klingensmith GJ, Williams DE, Ruggiero A, Liese AD, Bell RA, Waitzfelder BE, McKeown RE; SEARCH for Diabetes in Youth Study. Prevalence and correlates of depressed mood among youth with diabetes: the SEARCH for Diabetes in Youth study. Pediatrics 2006; 117: 1348-58.

16. Hood KK, Rausch JR, Dolan LM. Depressive symptoms predict change in glycemic control in adolescents with type 1 diabetes: rates, magnitude, and moderators of change. Pediatr Diabetes 2011; 12: 718-23.

17. Ly TT, Maahs DM, Rewers A, Dunger D, Oduwole A, Jones TW. Assessment and management of hypoglycemia in children and adolescents with diabetes. Pediatr Diabetes 2014; 15: 180-92.

18. Hopkins D, Lawrence IG, Mansell P, Thompson G, Amiel $\mathrm{S}$, Heller S. Improved biomedical and psychological outcomes one year after structured education in flexible insulin therapy for people with type 1 diabetes mellitus: the UK DAFNE experience. Diabetes Care 2012; 35: $1638-42$. 